\title{
Polycystic ovarian morphology in Thai women of reproductive age with polycystic ovary syndrome
}

\author{
Thanyarat Wongwananuruk ${ }^{\oplus}$, Panicha Chantrapanichkul*๑ ${ }^{*}$, Vichuta Unalome, \\ Suchada Indhavivadhana ${ }^{\oplus}$, Manee Rattanachaiyanont ${ }^{\oplus}$, Kitirat Techatraisak ${ }^{\oplus}$, \\ Surasak Angsuwathana ${ }^{\circledR}$, Kittayaporn Silprasit
}

\begin{abstract}
Background: Advancements in ultrasound technology have facilitated identifying polycystic ovarian morphology (PCOM) in women with and without polycystic ovary syndrome (PCOS), but it still has limitations due to follicle counting methods and variation of phenotypes according to ethnicity. Ethnicity-specific ovarian morphology may help to establish ethnicity-specific follicle count cut points for defining PCOM in women with PCOS.

Objectives: To investigate the prevalence and factors associated with PCOM in Thai women of a reproductive age with PCOS.

Methods: This prospective cross-sectional study was conducted in our gynecology department at a tertiary teaching hospital from February 2016 to May 2017. We included women with PCOS, who were measured for weight, height, waist circumference, and blood pressure. Blood samples were taken to measure fasting blood glucose, lipid profile, testosterone level, and $2 \mathrm{~h}$ post-load $75 \mathrm{~g}$ oral glucose tolerance test (OGTT). Transvaginal or transrectal sonography was performed to evaluate their ovaries.

Results: All 143 patient participants we included had oligomenorrhea, 77.6\% of them had acne, and 64.3\% hirsutism. Their average total testosterone level was $0.47 \pm 0.10 \mathrm{ng} / \mathrm{mL}$. The prevalence of PCOM was $55.2 \%$. The proportions of PCOM diagnosed by ovarian follicle and ovarian volume criteria were $36.4 \%$ and $42.0 \%$, respectively. There were $20.0 \pm 9.5$ follicles per ovary, $8.3 \pm 3.1$ follicles per cross section, and the mean ovarian volume was $7.9 \pm 3.0 \mathrm{~mL}$. Conclusion: The overall prevalence of PCOM in Thai women of reproductive age with PCOS was 55.2\%. Our univariate analysis found no factors significantly associated with PCOM.
\end{abstract}

Keywords: female; polycystic ovary syndrome; reproduction; Thai; ultrasonography

Polycystic ovary syndrome (PCOS) is one of the most common endocrinopathies, with a prevalence of approximately $10 \%$ among women of a reproductive age [1]. Insulin resistance (IR) is considered a main feature of women with PCOS. Consequently, these patients are at a higher risk of having type 2 diabetes mellitus (DM) and metabolic syndrome [2-6]. Early diagnosis of PCOS may facilitate early detection of metabolic disorders, which may help to prevent long-term metabolic sequelae. The etiology of PCOS remains unknown. Diagnosis of PCOS is currently based on the Revised Rotterdam Criteria

*Correspondence to: Panicha Chantrapanichkul, Gynecologic Endocrinology Unit, Department of Obstetrics and Gynecology, Faculty of Medicine Siriraj Hospital, Mahidol University, Bangkok 10700, Thailand, e-mail: panicha.cha@mahidol.ac.th Department of Obstetrics and Gynecology, Faculty of Medicine Siriraj Hospital, Mahidol University, Bangkok, Thailand

¿ Open Access. ๑ 2020 Wongwananuruk et al., published by Sciendo. (cc)BY-NC-ND This work is licensed under the Creative Commons Attribution NonCommercial-NoDerivatives 4.0 License. 
(2003) [7], which requires the presence of at least 2 of the following 3 criteria: (1) oligomenorrhea and/or amenorrhea, (2) clinical and/or biochemical hyperandrogenism, and/or (3) ultrasonographic evidence of polycystic ovarian morphology (PCOM). PCOM is defined as the presence of at least 12 follicles in cross-sectional plan measuring $2-9 \mathrm{~mm}$ in diameter and/ or volume of either ovary greater than $10 \mathrm{~mL}$. Other causes of chronic anovulation must be excluded before making a diagnosis of PCOS.

Advancements in ultrasound technology have facilitated identifying PCOM in women with and without PCOS $[8,9]$. Evidence found by many studies suggests that the threshold value of 12 or more follicles per cross section plane may need to be revised. In 2014, the Androgen Excess and Polycystic Ovary Syndrome Society (AEPCOS) conducted a systematic review to validate a new threshold value of follicle number per ovary (FNPO) for defining PCOM. The society task force issued a revised recommendation that at least 25 follicles should be used as a new threshold for defining PCOM [10]. Even with the new FNPO threshold for diagnosing PCOM, ultrasonography has limitations [11]. These limitations include differences in follicle counting methods, observer variability regarding the number of follicles, the impact of imaging technology on follicle count variability, and variation in phenotypes and endocrinology among different ethnic groups. Ethnicity-specific ovarian morphology in PCOS women may help to establish ethnicity-specific follicle count cut points for defining PCOM in PCOS women.

Accordingly, the primary aim of this study was to determine the prevalence of PCOM in Thai women of reproductive age with PCOS. The secondary objective was to identify factors associated with PCOM.

\section{Methods}

This prospective cross-sectional study in women diagnosed with PCOS was conducted at the Gynecologic Endocrinology Unit, Department of Obstetrics and Gynecology, Faculty of Medicine Siriraj Hospital, Mahidol University, Bangkok, Thailand, from February 2016 to May 2017. The study's protocol was approved by the Siriraj Institutional Review Board (COA Si049/2016), and written informed consent was obtained from all participants. The study was conducted in full compliance with international guidelines for human research protection, such as the Declaration of Helsinki, the Belmont Report, CIOMS Guidelines, and the International Conference on Harmonization in Good Clinical Practice (ICH-GCP) and their contemporary amendments. The present study is reported according to STROBE cross-sectional reporting guidelines [12].

Thai women with PCOS aged 18-45 years were included. PCOS was diagnosed according to the Revised Rotterdam Criteria 2003 [7]. Oligomenorrhea was defined as menstrual cycle length of more than 35 days or having menstruation fewer than 10 cycles per year. Amenorrhea was defined as the absence of menstrual bleeding for more than 6 months or 3 cycles. The presence of acne, hirsutism, male androgenetic alopecia (MAA), or virilization defined clinical hyperandrogenism. Acne was evaluated using the criteria recommended by the Dermatological Society of Thailand 2011 [13]. Acne severity was graded as follows: (1) mild acne: "presence of comedones and/or more than 10 papules or pustules"; (2) moderate acne: "more than 10 papules or pustules, and/or less than 5 nodules"; and (3) severe acne: "numerous papules, pustules, and/or nodules". In the present study, hirsutism was assessed according to the modified Ferriman-Gallway scoring (mFGs) system, with a cutoff score for diagnosis of at least 5 [14-17]. The Ludwig scale was used to categorize male androgenetic alopecia as mild, moderate, or severe. Biochemical evidence of hyperandrogenemia was defined as a serum total testosterone level $>0.8 \mathrm{ng} / \mathrm{mL}$ [18]. Women who had undergone surgery on either one or both ovaries, who were pregnant at the time of study enrollment, and/or who received hormone or steroid treatment within 3 months before the invitation to enroll in the present study were excluded.

Patient history was taken, including menstrual history, underlying disease, and current medication. Physical examination was conducted to measure vital signs, height, weight and waist circumference, mFGS or other signs of hyperandrogenism. All participants received a pelvic ultrasonographic scan, which was followed by a venous phlebotomy.

Pelvic ultrasonography was performed by using either a transvaginal approach (TVS) or transrectal approach (TRS), and the scan was performed by 2 ultrasonographers to evaluate for FNPO, follicle number per cross-section (FNPS), and ovarian volume $(\mathrm{OV})$. A TRS was performed in virgin women. Participants were evaluated during the follicular phase or the anovulatory period. Pelvic ultrasonography was performed using an Aloka ProSound Alpha 6 system (Hitachi Aloka Medical, Mitaka, Japan) with a $7.5 \mathrm{MHz}$ transvaginal or transrectal probe. Ovaries were evaluated starting from the inner to the outer border. If a dominant follicle, a corpus luteum, or some other abnormal ovarian cyst was detected by pelvic sonography, then participants were excluded. The length and width of the ovary were measured at the plane that demonstrated the most number of follicles. The transducer 
probe was then rotated $90^{\circ}$ to measure the thickness of the ovary. OV was calculated using a formula for a prolate ellipsoid $(0.5 \times$ length $\times$ width $\times$ thickness $)$. Follicle size was calculated by taking the average of 2 perpendicular diameters, and the follicles with a diameter of $2-9 \mathrm{~mm}$ were counted. In each ovary, the FNPS was measured in the ovarian plane containing the maximal number of follicles. The FNPO was measured by counting the total number follicles in the entire ovary, during slow and continuous scanning from one border to the other. Variables for both ovaries were recorded, and the highest values of FNPO, FNPS, and OV were used for analysis.

FNPO, FNPS, and OV were examined by 2 ultrasonographers to assess interobserver reliability. According to a previous study, intraclass correlation coefficient analysis reported an interobserver agreement of 0.94 for FNPS and 0.84 for FNPO [18], whereas interobserver reliability was 0.945 for FNPS and 0.959 for FNPO. Intraobserver reliability was 0.986 for the first sonographer and 0.981 for the second.

Blood samples were collected on the same day the pelvic scan was performed. After a $12 \mathrm{~h}$ overnight fast, blood was collected twice. The first sample was taken between 8:00 and 10:00 AM to measure the patient's metabolic profile (glucose, insulin, cholesterol, triglycerides, high-density lipoprotein [HDL], low-density lipoprotein [LDL], and total testosterone). The second sample was taken $2 \mathrm{~h}$ after administration of a $75 \mathrm{~g}$ glucose loading to determine their glucose and insulin levels.

All blood samples were sent to the laboratory unit of the Department of Clinical Pathology, Faculty of Medicine Siriraj Hospital, Mahidol University, for analysis. Total testosterone, HDL, LDL, cholesterol, triglycerides, and glucose assays were assayed on a Modular P800 Chemistry Analyzer (Roche Diagnostics). Insulin and total testosterone were assayed on a Modular E170 Modular Analytics Immunoassay Analyzer (Roche Diagnostics). All methods had inter- and intra-assay coefficients of variation $(\mathrm{CV})<5 \%$.

Patients with a body mass index (BMI) of $\geq 25 \mathrm{~kg} / \mathrm{m}^{2}$ were classified as obese according to the criteria set by the International Association for the Study of Obesity and the International Obesity Task Force 2000 [19]. Central obesity was defined as waist circumference (WC) $\geq 80 \mathrm{~cm}$ according to the International Diabetes Federation [6]. A blood pressure reading of $\geq 140 / 90 \mathrm{mmHg}$ was defined as hypertension according to the criteria set by the World Health Organization/International Society of Hypertension Writing Group (WHO/ISH) 2003 [20]. A serum level of total cholesterol $\geq 200 \mathrm{mg} / \mathrm{dL}$, or LDL $\geq 160 \mathrm{mg} / \mathrm{dL}$, or HDL $<50 \mathrm{mg} / \mathrm{dL}$, or triglycerides $\geq 150 \mathrm{mg} / \mathrm{dL}$ was defined as dyslipidemia according to the criteria set by the National Cholesterol Education Program Adult Treatment (NCEP ATP) III [21]. Glucose tolerance was evaluated using a $75 \mathrm{~g}$ oral glucose tolerance test (OGTT) according to the American Diabetes Association (ADA) [22]. Abnormal OGTT was classified as follows: (i) impaired fasting glucose (IFG) (i.e., fasting glucose $>100$ and $<126 \mathrm{mg} / \mathrm{dL}$ ), (ii) impaired glucose tolerance (IGT) (i.e., $2 \mathrm{~h}$ glucose $>140$ and $<200 \mathrm{mg} / \mathrm{dL}$ ), and (iii) type $2 \mathrm{DM}$ (fasting blood glucose $\geq 126 \mathrm{mg} / \mathrm{dL}$ and/ or $2 \mathrm{~h}$ glucose $\geq 200 \mathrm{mg} / \mathrm{dL}$ ). In the present study, IFG, IGT, and type $2 \mathrm{DM}$ were included in the summation of abnormal OGTT.

\section{Statistical analyses}

Statistical analyses were performed using the Statistical Package for the Social Sciences SPSS for Windows (version 14). Descriptive statistics were used to describe participant characteristics. Data are presented as number, percentage, or mean \pm standard deviation (SD). A Student $t$ test and MannWhitney $U$ test were used for univariate analysis for continuous data, and $\chi^{2}$ and Fisher exact tests were used for categorical data. No multivariate model was employed in this study, because univariate analysis revealed no significantly associated factors. $P<0.05$ is regarded as significant.

\section{Results}

We enrolled 143 Thai women with PCOS. Table 1 provides baseline demographic and other characteristics of the patients. All study participants had clinical oligo/amenorrhea and hyperandrogenism. The 2 most commonly presenting symptoms of clinical hyperandrogenism were acne and hirsutism. Half of all participants had dyslipidemia, and the average total testosterone level was $0.47 \pm 0.10 \mathrm{ng} / \mathrm{mL}$.

The ovarian morphology of 143 Thai women with PCOS is provided in Table 2.

Demographic, anthropometric, metabolic, and hormonal characteristics of 143 Thai women with PCOS are compared between those with and without PCOM in Table 3. There were no significant differences between groups for any of the parameters evaluated.

Univariate analysis for factors significantly associated with PCOM is shown in Table 4. This analysis revealed no significant associations between PCOM and any of the investigated factors, including obesity, clinical hyperandrogenism, hyperandrogenemia, glucose intolerance, or dyslipidemia. 
Table 1. Baseline demographic and clinical characteristics of 143 Thai women with PCOS

\begin{tabular}{lc}
\hline Demographic or characteristic & $\begin{array}{c}\text { Mean } \pm \text { standard deviation } \\
\text { or } \mathbf{n}(\%)\end{array}$ \\
\hline Age (years) & $25.2 \pm 5.3$ \\
Body mass index $\left(\mathrm{kg} / \mathrm{m}^{2}\right)$ & $24.3 \pm 6.0$ \\
$<25$ & $86(60.1)$ \\
$\geq 25$ & $57(39.9)$ \\
Waist circumference $(\mathrm{cm})$ & $79.6 \pm 13.8$ \\
Parity $\geq 1$ & $10(4.9)$ \\
Systolic blood pressure $(\mathrm{mmHg})$ & $115.3 \pm 15.9$ \\
Diastolic blood pressure $(\mathrm{mmHg})$ & $70.7 \pm 12.5$ \\
Clinical presentation & $143(100)$ \\
Oligomenorrhea & $143(100)$ \\
Hyperandrogenism & $111(77.6)$ \\
Acne & $24(16.8)$ \\
Alopecia & $92(64.3)$ \\
Hirsutism & \\
Biochemical investigations & $83.9 \pm 11.4$ \\
Fasting blood sugar (mg/dL) & $112.6 \pm 38.2$ \\
2 h 75 g OGTT (mg/dL) & $23(16.1)$ \\
Abnormal OGTT & $8(5.6)$ \\
Diabetes mellitus & $22(15.4)$ \\
Impaired glucose tolerance & $72(50.3)$ \\
Dyslipidemia & $194.8 \pm 33.9$ \\
Cholesterol (mg/dL) & $89.4 \pm 49.5$ \\
Triglycerides (mg/dL) & $61.3 \pm 17.8$ \\
High-density lipoprotein $(\mathrm{mg} / \mathrm{dL})$ & $115.1 \pm 33.9$ \\
Low-density lipoprotein $(\mathrm{mg} / \mathrm{dL})$ & $0.47 \pm 0.10$ \\
Total testosterone $(\mathrm{ng} / \mathrm{mL})$ & \\
\hline PCOS, polycystic ovary syndrome; OGTT, oral glucose tolerance test. \\
\end{tabular}

Table 2. Ovarian morphology of 143 Thai women with polycystic ovary syndrome

\begin{tabular}{lc}
\hline Ovarian morphology & $\begin{array}{c}\text { Mean } \pm \text { standard } \\
\text { deviation or } \mathbf{n}(\%)\end{array}$ \\
\hline PCOM & $79(55.2)$ \\
PCOM by ovarian follicle & $52(36.4)$ \\
PCOM by ovarian volume & $60(42.0)$ \\
Follicle number per ovary & $20.0 \pm 9.5$ \\
Follicle number per cross section & $8.3 \pm 3.1$ \\
Ovarian volume $(\mathrm{mL})$ & $7.9 \pm 3.0$ \\
\hline
\end{tabular}

PCOM, Polycystic ovarian morphology.

\section{Discussion}

The 55.2\% prevalence of PCOM found in this study differs greatly from the $28 \%$ prevalence reported from a previous meta-analysis [23] that included 12 studies (5 from Europe, 4 from Asia, 2 from America, and 1 from Australia). However, the subgroups in the previous meta-analysis were various (Europe, 40\%; America, 13\%; Asia, 21\%; Australia, 38\%). The meta-analysis found the prevalence of PCOM in PCOS to be raised in people of European ancestry with obesity and hyperandrogenism. In the present study, about $50 \%$ of women in the PCOM group had obesity, hyperandrogenism, and abnormal OGTT. Huang et al. reported a $92.9 \%$ prevalence of PCOM in Asian women with PCOS and 69.9\% in Middle Eastern women with PCOS [23]. A previous study of Thai women with PCOS found a prevalence of PCOM of $76.9 \%$ [24]. The prevalence of PCOM was higher in that study due to variations in the characteristics of the study population. The patients in the present study were younger and less obese than the population in the previous Thai study. It may then be suggested that these 2 factors influence endocrinology and ovarian morphology in PCOS women.

Welt et al. reported a larger OV and number of follicles in African American women and women with European ancestry than in Asian women [25]. In French women, Dewailley et al. found an FNPO of 19 follicles and OV of $7 \mathrm{~mL}$ [26]. Lujan et al. studied Canadian and American women with PCOS and found FNPO of 38 follicles, FNPS of 10 follicles, and $\mathrm{OV}$ of $13 \mathrm{~mL}$ [17]. A study of Asian women by Han et al. found an FNPO of 14 follicles and an $\mathrm{OV}$ of $7 \mathrm{~mL}$ [27]. The present study found an FNPO of $20.0 \pm 9.5$ follicles, an FNPS of $8.3 \pm 3.1$ follicles, and an ovarian volume of $7.9 \pm 3.0 \mathrm{~mL}$. The presence of phenotypic differences among various populations suggests genetic variability, and/or genetic or environmental modifiers of the PCOS phenotype in various ethnicities. Therefore, we were unable to identify a definite PCOM cut-point in women with PCOS because of variations in their phenotype, metabolic abnormality, endocrinology, genetics, and variations in follicle counting methods and methods of measurement by ultrasonography.

The International PCOS Networks Recommendation 2018 [28] suggests using pelvic ultrasonography to detect PCOM, which could be used with either a transabdominal or transvaginal approach. Transabdominal ultrasound is best focused on an ovarian volume having a cutoff of $10 \mathrm{~mL}$ or higher, while a transvaginal approach could be used for either follicle count or volume. The frequency of vaginal transducer should be high to provide good resolution. An automated volume calculation program in some ultrasound machines could be used to determine the number of follicles per ovary, per cross section, and ovarian volume. However, this program might not be available in every hospital, especially in regions having limited resources. 
Table 3. Demographic, anthropometric, metabolic, and hormonal characteristics of 143 Thai women with PCOS compared between those with and without PCOM

\begin{tabular}{|c|c|c|c|c|}
\hline Characteristic & Total & No PCOM & PCOM & $P$ \\
\hline Age (years) & $25.2 \pm 5.3$ & $25.4 \pm 5.9$ & $25.1 \pm 4.9$ & 0.70 \\
\hline Body mass index $\left(\mathrm{kg} / \mathrm{m}^{2}\right)$ & $24.2 \pm 6.1$ & $25.3 \pm 7.0$ & $23.4 \pm 5.1$ & 0.050 \\
\hline Waist circumference $(\mathrm{cm})$ & $79.5 \pm 13.9$ & $82.0 \pm 15.2$ & $77.4 \pm 12.4$ & 0.050 \\
\hline Hirsutism & 92 & $44(47.8)$ & $48(52.2)$ & 0.38 \\
\hline Acne & 111 & $51(45.9)$ & $60(54.1)$ & 0.69 \\
\hline Alopecia & 24 & $7(29.2)$ & $17(70.8)$ & 0.12 \\
\hline Fasting blood sugar (mg/dL) & $84.0 \pm 11.4$ & $84.9 \pm 14.7$ & $83.3 \pm 7.8$ & 0.39 \\
\hline 2 h 75 g OGTT (mg/dL) & $112.4 \pm 38.0$ & $114.6 \pm 35.1$ & $110.6 \pm 40.4$ & 0.52 \\
\hline Cholesterol (mg/dL) & $195.0 \pm 34.0$ & $192.6 \pm 32.5$ & $197.1 \pm 35.3$ & 0.43 \\
\hline Triglycerides (mg/dL) & $88.0 \pm 48.75$ & $86.7 \pm 44.9$ & $90.7 \pm 51.9$ & 0.62 \\
\hline Low-density lipoprotein (mg/dL) & $115.3 \pm 34.1$ & $115.6 \pm 33.7$ & $115.1 \pm 34.7$ & 0.93 \\
\hline High-density lipoprotein (mg/dL) & $61.6 \pm 17.8$ & $59.4 \pm 17.6$ & $63.4 \pm 17.9$ & 0.17 \\
\hline Fasting insulin $(\mu \mathrm{IU} / \mathrm{mL})$ & $12.6 \pm 10.9$ & $12.5 \pm 9.3$ & $12.6 \pm 12.1$ & 0.29 \\
\hline Albumin (g/dL) & $4.54 \pm 0.26$ & $4.48 \pm 0.28$ & $4.59 \pm 0.24$ & 0.44 \\
\hline Testosterone (ng/mL) & $0.47 \pm 0.18$ & $0.41 \pm 0.17$ & $0.52 \pm 0.18$ & 0.43 \\
\hline
\end{tabular}

OGTT, oral glucose tolerance test; PCOM, polycystic ovarian morphology; PCOS, polycystic ovary syndrome.

$P<0.05$ is considered significant.

Data presented as number, number and percentage, or mean \pm standard deviation.

Table 4. Univariate analysis for factors significantly associated with PCOM

\begin{tabular}{lccc}
\hline Factors & Patients & Patients with PCOM n (\%) & P \\
\hline Age $\geq 30$ years & 26 & $12(46.2)$ & 0.30 \\
Obesity & 57 & $28(49.1)$ & 0.30 \\
Central obesity & 62 & $29(46.8)$ & 0.07 \\
Hypertension & 13 & $6(46.2)$ & 0.49 \\
Acne & 111 & $60(54.1)$ & 0.59 \\
Hirsutism & 92 & $48(52.2)$ & 0.32 \\
Alopecia & 24 & $17(70.8)$ & 0.09 \\
Abnormal $75 \mathrm{~g}$ OGTT & 23 & $12(52.2)$ & 0.75 \\
Diabetes mellitus & 8 & $5(62.5)$ & 0.73 \\
Triglycerides $\geq 150 \mathrm{mg} / \mathrm{dL}$ & 17 & $9(52.9)$ & 0.84 \\
Cholesterol $\geq 200 \mathrm{mg} / \mathrm{dL}$ & 61 & $35(57.4)$ & 0.66 \\
High-density lipoprotein $<40 \mathrm{mg} / \mathrm{dL}$ & 14 & $6(42.9)$ & 0.33 \\
Testosterone $\geq 0.8 \mathrm{ng} / \mathrm{mL}$ & 8 & $6(75.0)$ & 0.30 \\
\hline
\end{tabular}

PCOM, polycystic ovarian morphology; OGTT, oral glucose tolerance test.

$P<0.05$ is considered significant.

The main clinical presentation of PCOS is anovulation, and the major risk factors for anovulation are obesity, insulin resistance, and hyperandrogenism. The pathophysiology of PCOM disease is not precisely known. To our knowledge, this is the first study to investigate the factors significantly associated with PCOM, apart from the risk factors for anovulation. However, we found no significant association between any of the factors evaluated and PCOM, which suggests a complex pathophysiology of PCOM and possibly underpowered sample size.

A strength of this study is the presentation of new data for PCOM in Thai women of reproductive age with PCOS, including prevalence, ovarian morphology, and factors associated with PCOM. Our study has several limitations, including 
its small sample size and single-center design. Furthermore, some factors might affect ultrasonographic findings of PCOM, such as variability in population, ultrasound machine, and skill of technicians. Further prospective studies in a larger study population from multiple centers, and the other methods using evaluation of ovaries, for instance, an automated volume calculated program, $\mathrm{AMH}$, or other serum markers are warranted to confirm these findings and to elucidate PCOM further in Thai women with PCOS.

\section{Conclusion}

The total prevalence of PCOM among Thai women of reproductive age with PCOS was $55.2 \%$. Our univariate analysis found no factors significantly associated with PCOM.

Author contributions. TW and VU contributed to the conception and design of the study. KS and PC acquired the data and TW, SI, MR, KT, and SA analyzed it. TW, VU, KS, and PC drafted the manuscript and SI, MR, KT, and SA critically revised it. All authors approved the final version submitted for publication and take responsibility for statements made in the published article.

Acknowledgments. The authors gratefully acknowledge the staff of the Gynecologic Endocrinology Unit, Department of Obstetrics and Gynecology, Faculty of Medicine, Siriraj Hospital, who participated in this study, and Julaporn Pooliam of the Division of Clinical Epidemiology, Department of Research and Development, Faculty of Medicine Siriraj Hospital, for assistance with statistical analysis. The authors express gratitude to the Japan Society of Obstetrics and Gynecology for allowing us to present an abstract of this work at the 70th Annual Congress of the Japan Society of Obstetrics and Gynecology, 2018 May 11-13, Sendai, Miyagi, Japan. International poster session ISP-23-1. Unalome V, Wongwananurak T. Polycystic ovarian morphology in reproductive-aged Thai women with polycystic ovary syndrome. J Obstet Gynaecol Res. 2018; 44:1578. The authors did not receive any specific grant for this research from any funding agency in the public, commercial, or not-for-profit sectors.

Conflicts of interest statement. Each author has completed and submitted an International Committee of Medical Journal Editors Uniform Disclosure Form for Potential Conflicts of Interest. None of the authors has any potential of actual conflict of interest to disclose in relation to the present article.
Data sharing statement. The data sets generated or analyzed during the present study are included in this published article. Further details are available from the corresponding author for noncommercial purposes on reasonable request after deidentification of data from any person whose data was included in the study.

\section{References}

[1] Norman RJ, Dewailly D, Legro RS, Hickey TE. Polycystic ovary syndrome. Lancet. 2007; 370(9588):685-97.

[2] Goodman NF, Cobin RH, Futterweit W, Glueck JS, Legro RS, Carmina E; American Association of Clinical Endocrinologists (AACE); et al. American Association of Clinical Endocrinologists, American College of Endocrinology, and Androgen Excess and PCOS Society Disease State clinical review: guide to the best practices in the evaluation and treatment of polycystic ovary syndrome - Part 1. Endocr Pract. 2015; 21:1291-300.

[3] Indhavivadhana S, Wongwananuruk T, Rattanachaiyanont $M$, Techatraisak K, Leerasiri P, Tanmahasamut P, Popijan M. Prevalence of metabolic syndrome in reproductive-aged polycystic ovary syndrome Thai women. J Med Assoc Thai. 2010; 93:653-60.

[4] Wongwananuruk $T$, Rattanachaiyanont $M$, Indhavivadhana $S$, Leerasiri P, Techatraisak K, Tanmahasamut P, et al. Prevalence and clinical predictors of insulin resistance in reproductive-aged thai women with polycystic ovary syndrome. Int J Endocrinol 2012; 2012:529184. doi: 10.1155/2012/529184

[5] Wongwananuruk $T$, Indhavivadhana S, Rattanachaiyanont M, Techatraisak K, Leerasiri P, Tanmahasamut P, et al. Characteristics of 250 reproductive-aged polycystic ovary syndrome Thai women at Siriraj Hospital. J Med Assoc Thai. 2010; 93:399-405.

[6] Alberti KGMM, Zimmet P, Shaw J. Metabolic syndrome-a new world-wide definition. A Consensus Statement from the International Diabetes Federation. Diabet Med. 2006; 23:469-80.

[7] Rotterdam ESHRE/ASRM-Sponsored PCOS consensus workshop group. Revised 2003 consensus on diagnostic criteria and long-term health risks related to polycystic ovary syndrome (PCOS). Hum Reprod. 2004; 19:41-7.

[8] Johnstone EB, Rosen MP, Neril R, Trevithick D, Sternfeld B, Murphy R, et al. The polycystic ovary post-Rotterdam: a common, age-dependent finding in ovulatory women without metabolic significance. J Clin Endocrinol Metab. 2010; 95:4965-72.

[9] Kristensen SL, Ramlau-Hansen CH, Ernst E, Olsen SF, Bonde JP, Vested A, Toft G. A very large proportion of young Danish women have polycystic ovaries: is a revision of the Rotterdam criteria needed? Hum Reprod 2010; 25(12):3117-22.

[10] Dewailly D, Lujan ME, Carmina E, Cedars MI, Laven J, Norman RJ, Escobar-Morreale HF. Definition and significance of polycystic ovarian morphology: a task force report from the Androgen Excess and Polycystic Ovary Syndrome Society. Hum Reprod Update 2014; 20:334-52

[11] Lie Fong S, Laven JSE, Duhamel A, Dewailly D. Polycystic ovarian morphology and the diagnosis of polycystic ovary syndrome: redefining threshold levels for follicle count and serum anti-Müllerian hormone using cluster analysis. Hum Reprod. 2017; 32:1723-31. 
[12] von Elm E, Altman DG, Egger M, Pocock SJ, Gotzsche PC, Vandenbroucke JP. The Strengthening the Reporting of Observational Studies in Epidemiology (STROBE) Statement: guidelines for reporting observational studies. PLoS Medicine. 2007; 4:1628-54.

[13] Dermatological Society of Thailand. Clinical Practice Guideline Acne. [Internet] 2010 [cited 2020 Mar 23]; Available from: http:// www.dst.or.th/files_news/Acne_2010.pdf [in Thai]

[14] Kim JJ, Chae SJ, Choi YM, Hwang SS, Hwang KR, Kim SM, et al. Assessment of hirsutism among Korean women: results of a randomly selected sample of women seeking pre-employment physical check-up. Hum Reprod. 2011; 26:214-20.

[15] Zhao X, Ni R, Li L, Mo Y, Huang J, Huang M, et al. Defining hirsutism in Chinese women: a cross-sectional study. Fertil Steril 2011; 96:792-6.

[16] Wong M, Zhao X, Hong Y, Yang D. Semiquantitative assessment of hirsutism in 850 PCOS patients and 2,988 controls in China. Endokrynol Pol. 2014; 65:365-70.

[17] Li R, Qiao J, Yang D, Li S, Lu S, Wu X, Wei Z. Epidemiology of hirsutism among women of reproductive age in the community: a simplified scoring system. Eur J Obstet Gynecol Reprod Biol. 2012; 163:165-9.

[18] Lujan ME, Jarrett BY, Brooks ED, Reines JK, Peppin AK, Muhn N, et al. Updated ultrasound criteria for polycystic ovary syndrome: reliable thresholds for elevated follicle population and ovarian volume. Hum Reprod. 2013; 28:1361-8.

[19] World Health Organization. Regional Office for the Western Pacific. The Asia-Pacific perspective: redefining obesity and its treatment. Sydney: Health Communications Australia; 2000. [cited 2020 May 23]. Available from: https://apps.who.int/iris/handle/10665/206936

[20] Whitworth JA; World Health Organization, International Society of Hypertension Writing Group. World Health Organization (WHO)/International Society of Hypertension (ISH) statement on management of hypertension. J Hypertens. 2003; 21:1983-92.
[21] National Cholesterol Education Program (NCEP) Expert Panel on Detection, Evaluation, and Treatment of High Blood Cholesterol in Adults (Adult Treatment Panel III). Third Report of the National Cholesterol Education Program (NCEP) Expert Panel on Detection, Evaluation, and Treatment of High Blood Cholesterol in Adults (Adult Treatment Panel III) final report. Circulation. 2002; 106:3143-421.

[22] American Diabetes Association. Diagnosis and classification of diabetes mellitus. Diabetes Care. 2014; 37(Suppl 1):S81-90.

[23] Huang Z, Yong E-L. Ethnic differences: is there an Asian phenotype for polycystic ovarian syndrome? Best Pract Res Clin Obstet Gynaecol. 2016; 37:46-55.

[24] Vutyavanich T, Khaniyao V, Wongtra-Ngan S, Sreshthaputra O, Sreshthaputra R, Piromlertamorn W. Clinical, endocrine and ultrasonographic features of polycystic ovary syndrome in Thai women. J Obstet Gynaecol Res. 2007; 33:677-80.

[25] Welt CK, Arason G, Gudmundsson JA, Adams J, Palsdottir H, Gudlaugsdottir G, et al. Defining constant versus variable phenotypic features of women with polycystic ovary syndrome using different ethnic groups and populations. J Clin Endocrinol Metab. 2006; 91:4361-8.

[26] Dewailly D, Gronier H, Poncelet E, Robin G, Leroy M, Pigny P, et al. Diagnosis of polycystic ovary syndrome (PCOS): revisiting the threshold values of follicle count on ultrasound and of the serum AMH level for the definition of polycystic ovaries. Hum Reprod. 2011; 26:3123-9.

[27] Han YS, Lee AR, Song HK, Choi JI, Kim JH, Kim MR, Kim MJ. Ovarian volume in Korean women with polycystic ovary syndrome and its related factors. J Menopausal Med. 2017; 23:25-31.

[28] Teede HJ, Misso ML, Costello MF, Dokras A, Laven J, Moran L. International PCOS Network. Recommendations from the international evidence-based guideline for the assessment and management of polycystic ovary syndrome. Clin Endocrinol (Oxf). $2018 ; 89: 251-68$. 